\title{
Discovery of Photocatalysts for Hydrogen Production
}

\author{
D. Brent MacQueen (PI) \\ SRI International \\ 333 Ravenswood Avenue \\ Menlo Park, CA 94025 \\ Phone (650) 859-5286 \\ FAX (650) 859-5286 \\ e-mail brent.macqueen@sri.com \\ Nobi Kambe and Timothy Jenks \\ NanoGram Corporation \\ 46774 Lakeview Boulevard \\ Fremont, CA 94532 \\ Phone (510) 407-3232 \\ E-mail kambe@nanogram.com \\ DOE Project Manager: Roxanne Garland \\ Phone (202) 586-7260 \\ E-mail roxanne.garland@ee.doe.gov \\ DOE Project Officer: Jill Gruber \\ 303-275-4961, jill.gruber@go.doe.gov \\ Contract Number: DE-FC36-01G011093
}




\section{TABLE OF CONTENTS}

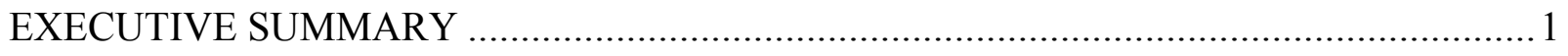

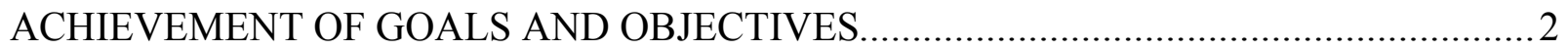

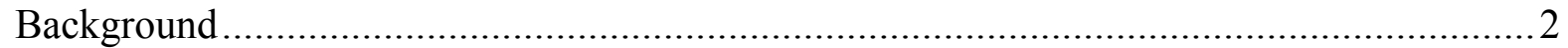

Progress on Development of Combinatorial Tools to Identify Semiconductors ...................... 3

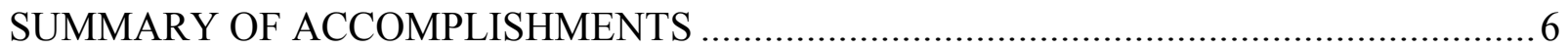

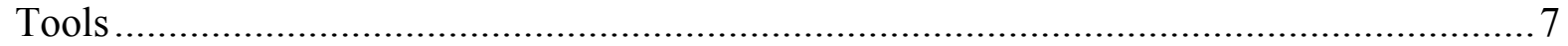

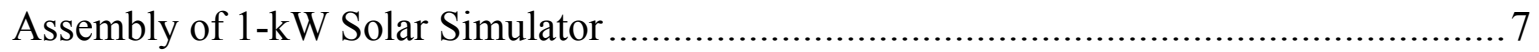

Calibration of Solar Simulator...................................................................................

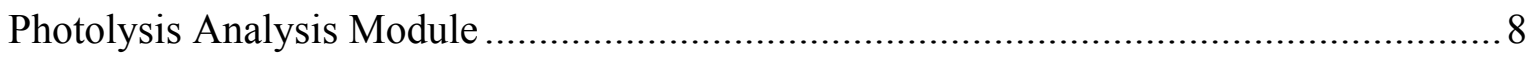

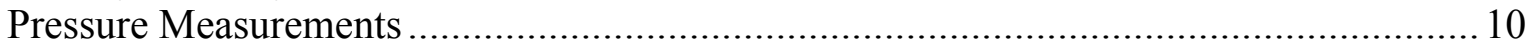

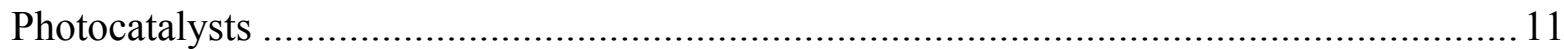

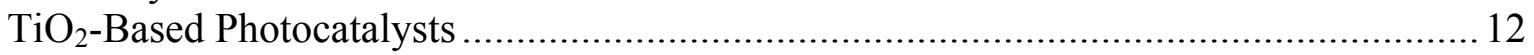

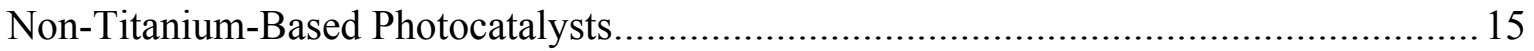

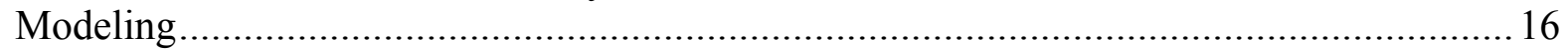

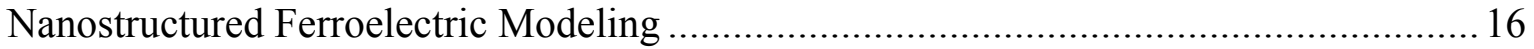

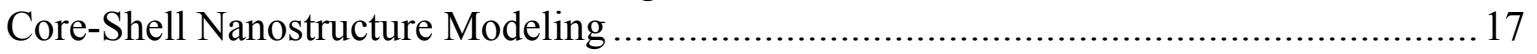

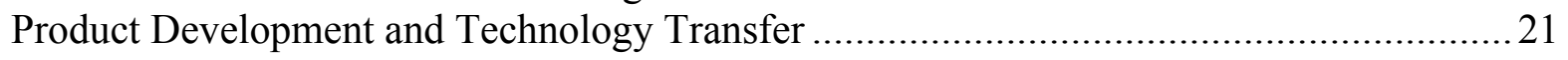

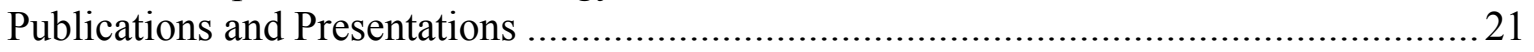

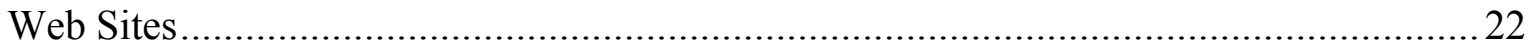

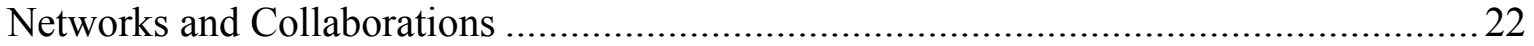

Technologies and Techniques...........................................................................22

Inventions/Patent Applications, Licensing Agreements .............................................. 22

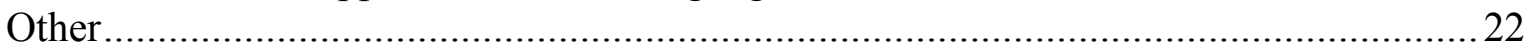

APPENDIX A: Core - Shell Nanorods for Efficient Photoelectrochemical Hydrogen

Production ERROR! BOOKMARK NOT DEFINED.

\section{LIST OF FIGURES}

Figure 1. Diagram depicting PEC water splitting process

Figure 2. Plot showing normalized hydrogen production as a function of surface area and by weight

Figure 3. Expanded view of 25-cell analysis module design

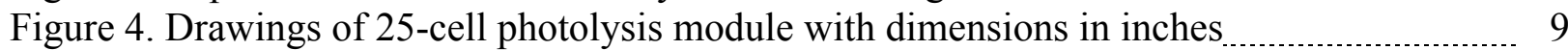

Figure 5. 25-Cell photolysis module with top open …….................................................... 9

Figure 6. Picture showing 25-cell photolysis module in operation $\quad 10$

Figure 7. Plot of raw data from 25-cell photolysis module _............................................. 10

Figure 8. NanoGram laser pyrolysis process

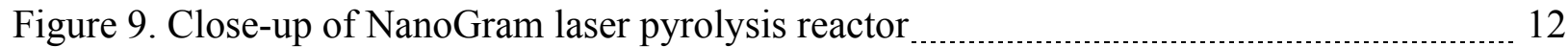

Figure 10. Solar spectrum (left) $(1 \mathrm{~mm}=1,000 \mathrm{~nm})$, and absorbance spectrum (right)

of several titania materials $\quad 14$

Figure 11. XRD pattern indicating formation of a mixed alkali $(\mathrm{Na} / \mathrm{K})$ indium oxide _................. 14

Figure 12. Absorbance spectrum of a niobate compared to a titania $\quad 15$ 
Figure 13. UV-Vis spectrum and XRD of (AgIn) $0.2 \mathrm{ZnS}$

Figure 14. Core-shell nano-rod structures indicating dimension definitions

Figure 15. Band alignment of the InP/ZnSe core/shell sphere. Red and black lines are for the valence and conduction bands, respectively

Figure 16. Spatial distribution of the HOMO (solid color) and LUMO (skeleton) indicating isolation

Figure 17. Integrated charge distribution in core for the HOMO (squares) and the LUMO

(circles) as a function of R1 in core-shell InP-CdS nanorods with fixed R=5 nm and L=2 nm 20

Figure 18. Suggested nano-rod core-shell structures and mechanism of charge separation

\section{LIST OF TABLES}

Table 1. Calibration Data for Four-Cell Block with 1 kW Xenon Lamp ..................................................................8

Table 2. Selected Data from Photolysis Experiments of Various $\mathrm{TiO}_{2}$ Samples....................................................13

\section{LIST OF ABBREVIATIONS}
ADC analog to digital
GHG greenhouse gas
PEC photoelectrochemical
PMMA poly(methylmethacrylate)
$\mathrm{PV} / \mathrm{EL}$ photovoltaic-driven electrolysis
XRD X-ray diffraction 


\section{EXECUTIVE SUMMARY}

It is well recognized that alternatives to fossil-fuel-based energy are needed due to supply, environmental, and national security issues. An alternative energy carrier being proposed is hydrogen. However, the primary method of producing hydrogen as fuel involves stripping the hydrogen from hydrocarbons in a process called steam reforming. This process generates significant quantities of $\mathrm{CO}_{2}$, an undesirable greenhouse gas. SRI and others have been working to develop and improve a process termed photoelectrochemical hydrogen production. This process has the potential to provide for economical and environmentally sound production of hydrogen by splitting water with sunlight using a semiconductor catalyst. However, the process, while proven, is currently not economical due to shortcomings in the semiconductor's material properties.

This project for DOE was designed to address these materials-related issues through a combination of high-throughput screening of semiconductor candidates and theoretical modeling of nanostructures. High-throughput screening is an effective and economical way to examine a large number of candidates and identify those worthy of further study. Unfortunately, in the course of this project, we discovered no semiconductor candidates that can meet the DOE's stringent requirements for an economically feasible photoelectrochemical process. However, some of our results indicated that several systems may have potential if further optimized. In particular, the published theoretical modeling work indicates that core-shell nanorod structures, if properly engineered, have the potential to overcome the shortfalls of current semiconductors. Although the synthesis of the designed core-shell nanorod structures proved to be beyond the current capabilities of our laboratories, recent advances in the synthesis of core-shell nanorod structures imply that the designed structures can be synthesized. SRI is confident that once these materials are made they will validate our models and lead to economical and environmentally friendly hydrogen from sunlight and water.

The high-throughput photolysis analysis module developed at SRI will also have utility in applications such as identifying catalysts for photo-assisted chemical detoxification, as well as non-photolytic applications such as hydrogen storage, which can take advantage of the ability of the analysis module to monitor pressure over time.

In summary this project has developed tools, both experimental and theoretical, that will, in time, have a direct impact on DOE's goal of developing economical and environmentally sound methods for producing hydrogen. While the results obtained with these tools in this project were somewhat disappointing, SRI is confident that they will play a significant role in the future development of this essential technology. Also, the photolysis analysis module has the potential to advance other technologies of interest to DOE, such as hydrogen storage and photo-assisted environmental remediation. 


\section{ACHIEVEMENT OF GOALS AND OBJECTIVES}

\section{Background}

The use of hydrogen as an energy carrier has been proposed as a means to alleviate the environmental issues related to the current dependency of the energy sector on fossil fuel resources. However, hydrogen production is currently based on technologies that rely on fossil fuels as either a feedstock, a methane reformer, or an energy source (water electrolysis using grid power). Photoelectrochemical (PEC) production of hydrogen using water as the feedstock, sunlight as the power source, and a semiconductor-based photocatalyst system has the potential to produce hydrogen without the generation of greenhouse gas (GHG).

PEC hydrogen production involves the direct utilization of photogenerated charge carriers at an electrode/electrolyte interface in the electrolysis of water to split it into hydrogen and oxygen. This project addressed materials-based issues that are discussed in DOE's "Hydrogen Posture Plan: An Integrated Research, Developemnt and Demonstration Plan" released in February 2004, and have identified as barriers to commercial implementation of PEC hydrogen production. The ability to generate hydrogen from the surface of a semiconductor utilizing water as the feedstock and sunlight as the energy source has been described as a "Holy Grail" of chemistry [A. J. Bard and M. A. Fox, Acc. Chem. Res. 28, 141 (1995)]. PEC hydrogen generation involves the direct utilization of photogenerated charge carriers (electrons and holes) in the splitting of water into hydrogen and oxygen. The successful implementation of a PEC source of hydrogen would contribute significantly to the reduction of $\mathrm{CO}_{2}$ generated and reduce the reliance of the United States on petroleum feed stocks for energy production and transportation.

In principal, PEC hydrogen production is analogous to a photovoltaic-driven electrolysis (PV/EL) process in that photogenerated charge carriers are generated in a semiconductor material and transferred to an electrode/electrolyte interface, which then facilitates the reduction/ oxidation of water. However, PEC is drastically different from PV/EL in that the current densities are lower in the PEC process, which reduces the over potential required to split water and makes this process more efficient. Also, with PEC there is no need for the electronics and electrolyzer required in PV/EL. A simplified depiction of the PEC process, shown in Figure 1, consists of light promoting a valence electron into the conduction band generating the charge carriers, which then migrate to the semiconductor/electrolyte interface and effect water splitting. As indicated, there is usually a catalyst (technically an electrocatalyst) that facilitates the electron transfer from the semiconductor into the electrolyte. 
Efficient PEC hydrogen production requires a suitable semiconductor photocatalyst that simultaneously satisfies four stringent requirements: 1) The semiconductor must have a band gap with value of $1.5-2.2 \mathrm{eV}$ to efficiently utilize the solar spectrum;

2) the conduction and valence band energies, or the LUMO and HOMO, of the materials must overlap the $\mathrm{H}_{2} / \mathrm{H}_{2} \mathrm{O}$ and $\mathrm{O}_{2} / \mathrm{H}_{2} \mathrm{O}$ redox potentials;

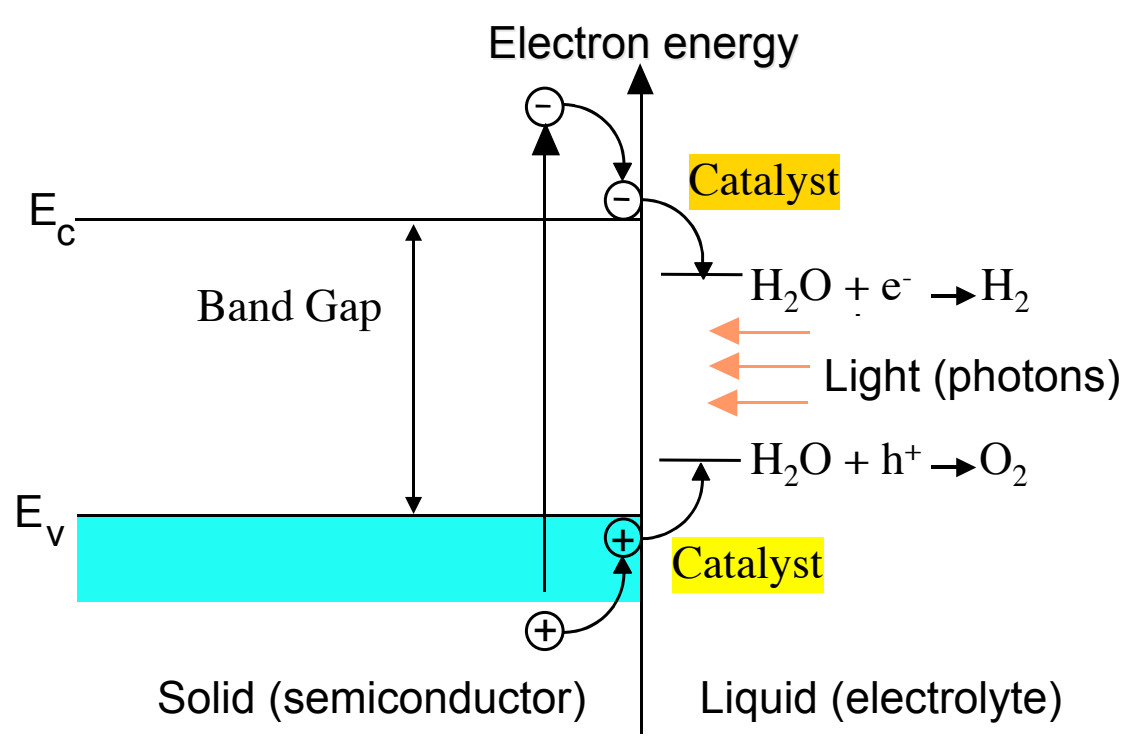

3 ) charge transfer across the

Figure 1. Diagram depicting PEC water-splitting process. material-liquid interface must be fast; and 4) the material surface must be chemically stable in the aqueous media under solar illumination. There are materials that satisfy some of these requirements; for example, $\mathrm{InGaP}_{2}$ has shown promising efficiencies but suffers from poor stability [O. Khaselev and J. Turner, J. Electrochem. Soc., 145, 3335-3339, 1998]. On the other hand, $\mathrm{TiO}_{2}$ is very stable and does exhibit efficiencies that would satisfy the established DOE Milestone for 2010 when illuminated with wavelengths of light greater than its bandgap of $3.2 \mathrm{eV}$. However, this bandgap is on the extreme high-energy side of the solar spectrum, which renders this material very inefficient at converting solar energy to hydrogen.

\section{Progress on Development of Combinatorial Tools to Identify Semiconductors}

This project was initiated to develop tools both experimental and, in later stages, theoretical, that will expedite the analysis of semiconductor candidates, with the overall goal being to identify for a more detailed analysis those semiconductor materials that satisfy the requirements for PEC hydrogen production. The key advancements this project provided to further the understanding of issues related to PEC hydrogen production were in the development of a tool to rapidly screen photocatalysts and the generation of theoretical models suggesting how nanostructures can overcome current obstacles to PEC hydrogen production. As the DOE Roadmap indicates, the barriers to PEC hydrogen production should be surmountable by discovery of improved semiconductor materials. To this end, SRI constructed a 25-cell photolysis module that can analyze a number of different semiconductor materials to quickly identify those materials with appropriate properties relevant to PEC hydrogen production that could then be characterized in more detail. Initial semiconductor materials analysis focused almost exclusively on nanoparticulate materials, and modifications thereof, supplied by NanoGram Corp., our partner on this effort. This effort, while reinforcing the potential beneficial efficiencies gained from 
nanoparticle photocatalysts as a result of increased surface area to volume ratio of nanoparticles, failed to identify a semiconductor material with suitable efficiency to warrant further detailed investigation in a PEC hydrogen production system. The effect of observed hydrogen production on particle size, and hence surface area, is shown in Figure 2. In looking at the hydrogen produced as a function of weight of catalyst, it would appear that as the particles get smaller the amount of hydrogen, on a per weight basis, is getting larger. However if one normalizes the data to surface area there is no observed difference in photocatalytic activity as a function of particle size. Subsequently, we investigated NanoGram materials modified

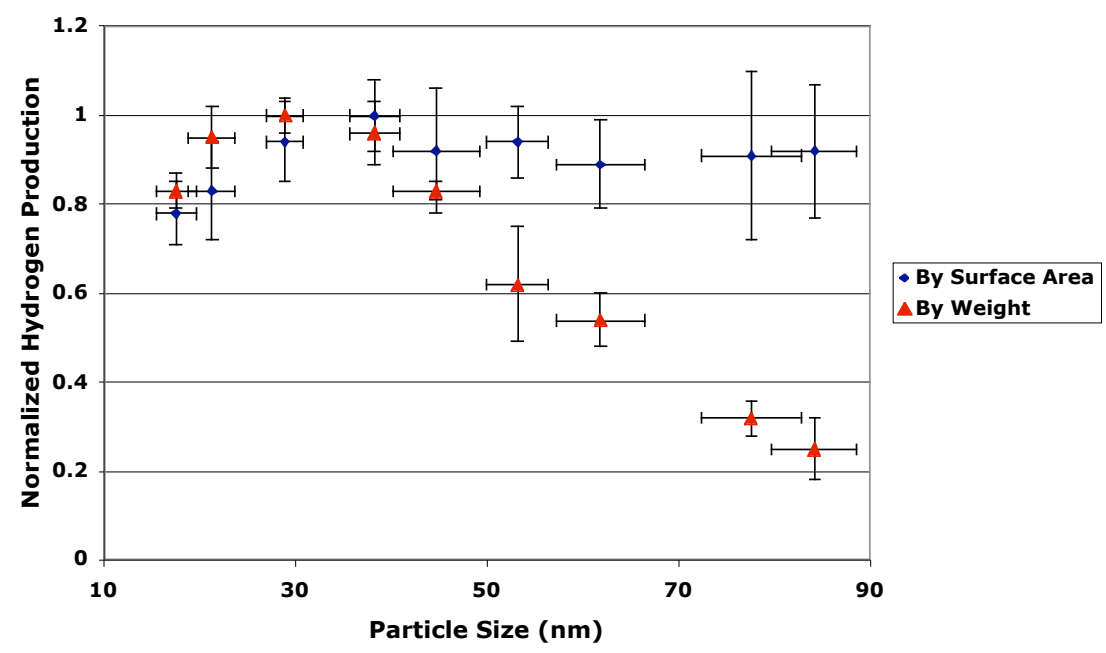
at SRI and materials generated exclusively at SRI. Initial materials selections were based on literature reports of semiconductor materials that absorbed more of the solar spectrum than $\mathrm{TiO} 2$, typically with some modification to either stoichiometry or processing conditions in addition to those published. The best results were obtained with systems based on TiOxNy's or $(\operatorname{AgIn})_{x} \mathrm{Zn}_{(1-x)} \mathrm{S}$, with the latter exhibiting the highest relative solar to hydrogen efficiency of all materials studied, but still well short of the projected efficiency (10\% solar to hydrogen) and stability (10 year lifetime) requirements for implementation of this material in a PEC hydrogen production system.

As a result of literature indicating the ability of core-shell nanoparticles to be engineered such that photogenerated charge carriers are separated and spatially isolated, indicating efficient charge separation, SRI initiated a modeling effort to determine if nanorod core-shell structures could be expected to behave in a similar fashion as the spherical materials. The modeling indicated that, indeed, core shell nanorod structures do have the potential ability to increase charge separation, which should overcome some of the mechanisms associated with charge recombination and photocorrosion of the semiconductor. Unfortunately we were unable to synthesize materials with the dimensions that the modeling indicated would be functional, and we were unable to verify the models. The main problem was getting an area of gold nanoparticles, the catalyst for InP rod growth, of the appropriate size and pitch. All of the materials we produced had dimensions greater than $100 \mathrm{~nm}$, well above the size needed. Recent 
advancements in the generation of nanoparticle catalyst arrays and in the growth of core-shell type materials do leave us optimistic that the predicted structures can be synthesized and will validate the models SRI has generated.

In summary, the goals of developing tools, both experimental and theoretical, have been achieved. The experimental tool was used to study a number of different semiconductor materials, however none of these materials provided evidence that they are suitable for use in a cost-effective PEC hydrogen production. The modeling has provided theoretical evidence that core-shell nanostructures may provide improved charge separation efficiencies and as such should improve efficiency of solar to hydrogen conversion as well as improve the photostability, and hence system lifetime, of the semiconductor toward photocorrosion. 


\section{SUMMARY OF ACCOMPLISHMENTS}

Energy production in the United States for utilization in sectors relevant to the DOE mission - industrial, residential and transportation - rely predominantly on fossil fuel-based resources whose continued use is questionable with respect to both supply and environmental sustainability. The development of hydrogen as an energy resource has been recently identified by DOE as a potential solution to the energy sectors' fossil fuel dependency. Current hydrogen production in the U.S. is based on stripping hydrogen from hydrocarbons, resulting in release of carbon dioxide, a greenhouse gas (GHG). To achieve the environmental benefits of the hydrogen as an energy carrier, sources of hydrogen need to be developed that do not rely on hydrocarbons, as either a direct, methane reformation, or indirect, water electrolysis using hydrocarbon generated electricity. Photoelectrochemical (PEC) production of hydrogen using water as the feedstock, sunlight as the power source and a semiconductor-based photocatalyst system has the potential to fulfill these goals. This technology involves the direct utilization of photogenerated charge carriers at a semiconductor electrolyte interface to split water into hydrogen and oxygen and has efficiency and potential cost advantages over other water electrolysis technologies. The generation of hydrogen from water and sunlight, PEC hydrogen production, would be a true renewable energy resource and is considered to be a "Holy Grail" [A. J. Bard and M. A. Fox, Acc. Chem. Res. 28, 141 (1995)] by some research scientists

PEC production of hydrogen is a proven technology being initially demonstrated by Honda in 1972 [A. Fujishima, K. Honda, Nature 238, p 37. 1972]. However, there are currently several barriers to its general implementation. DOE roadmaps have identified the barriers to PEC hydrogen production to be related to the properties of the semiconductor materials employed in the photocatalytic process. Specifically, to be commercially viable there is a need for more efficient utilization of the solar spectrum, a better match between the energetics of the photogenerated charge carriers to the required reduction and oxidation potentials of water, and improved long-term photostability of the semiconductor material. This project was initiated to synthesize nanoparticle semiconductor materials and to develop high throughput analysis methodologies to rapidly screen the materials in order to identify candidates for more extensive studies. SRI's partner in this project was a nanoparticulate semiconductor producer, NanoGram, whose particle synthetic process is scalable to $\mathrm{kg} / \mathrm{hour}$ quantities.

Perhaps the most significant accomplishment is in our modeling work, which indicates that developing nanostructures will give us the potential to solve the stability problems that have plagued the development of PEC hydrogen production. These developments indicate that, for example, the stability issues associated with most of the common semiconductor materials may be solved by the use of core shell structures. The key to this accomplishment is the finding that the lattice mismatch can be utilized to control the energetics of mixed semiconductor materials. The prevalent mechanism for the decomposition of the semiconductor material in PEC is the buildup of holes at the semiconductor electrolyte interface, resulting in the photocorrosion of 
semiconductor materials known to have the energetics appropriate for PEC hydrogen. A significant mechanism of photocorrosion is understood to be currently associated with disproportionate rates of consumption of electrons and holes at the semiconductor-electrolyte interface. The models developed indicate that, using the appropriate nanostructures, the rates of these reactions can be adjusted, or at least the removal of the excess charge carriers can be manipulated such that the stability of existing materials can be observed. The potential of core shell type materials was not realized until late in the project, and efforts to prove this hypothesis were not successful. However, the recent increase in the development of synthetic protocols for the generation of core-shell-type structures is an encouraging sign that experimental validation of the models will be forthcoming.

This summary is divided into three sections: Tools, Photocatalysts, and Modeling.

\section{Tools}

\section{Assembly of 1-kW Solar Simulator}

A 1-kW solar simulator was assembled by coupling an Oriel 1-kW xenon lamp with its associated housing and power supply to a full reflecting front surface mirror through a condenser, water filter, and electronic shutter (all from Oriel). This assembly was positioned on the floor below a bench top with a circular opening through which the light projects onto the bottom of a reaction cell. The light beam is reflected upward from the mirror through a beam turner and through first a $7.6 \mathrm{~cm}$ lens and then through a secondary $20 \mathrm{~cm}(8 \mathrm{in})$ collimating lens to impinge on a flat borosilicate plate on the bench top opening. At this point, all light below $290 \mathrm{~nm}$ and most light above $900 \mathrm{~nm}$ has been removed through the combination of the water filter and borosilicate plate.

\section{Calibration of Solar Simulator}

To determine both the intensity and intensity distribution of simulated sunlight at the top of the borosilicate plate, two kinds of light intensity measurements were made using both irradiance measurements with an International Light radiometer (Model IL1400A) and a solar active probe (SEL033) and photon intensity measurements with a chemical actinometer added to each of the four cells.

The xenon lamp was turned on and warmed up for 30-60 minutes before any measurements were made. The poly(methylmethacrylate) (PMMA) cell block was centered on top of the Pyrex sheet and fiduciary marks were made to the block and sheet to ensure reproducible positioning in the future. Cells were numbered 1-2 top and 3-4 bottom. An arrow on the block points to the top.

Radiometry on the cells was performed with a 1-mm pinhole aperture in the solar probe. The radiometer was zeroed and the probe then centered over each of the four cells while power readings were made (in $\mathrm{mW} / \mathrm{cm}^{2}$ ). The probe was scanned a few millimeters to the right, left, top and bottom of each cell to get a range of irradiance readings in each cell. The values varied a 
little with an average of $51( \pm 1-2) \mathrm{mW} / \mathrm{cm}^{2}$ over the face of the cells. These values are shown in Table 1 below.

Table 1. Calibration Data for Four-Cell Block with $1 \mathrm{~kW}$ Xenon Lamp

\begin{tabular}{|c|c|c|c|c|}
\hline Cell \# & 1 & 2 & 3 & 4 \\
\hline Power $\mathrm{mW} / \mathrm{cm}^{2} 1$ st meas. & $48+1$ & $52+0.5$ & $53+1$ & $51.5+1.5$ \\
\hline Power $\mathrm{mW} / \mathrm{cm}^{2} 2 \mathrm{nd}$ meas & $46+1$ & $51+1$ & $53+1$ & $51+\overline{0.5}$ \\
\hline $\begin{array}{l}\text { Rate constants for photolysis } \\
\text { with AZB in } \mathrm{Ms}^{-1}\end{array}$ & $3.20 \times 10^{-4}$ & $3.45 \times 10^{-4}$ & $3.74 \times 10^{-4}$ & $3.63 \times 10^{-4}$ \\
\hline
\end{tabular}

Actinometry was performed on the cell block using four 3-mL aliquots of $100 \square \mathrm{M}$ azoxybenzene in EtOH added to the cells for 3-, 7-, 11- and 16-minute exposures to the xenon lamp. This volume of AZB ensures that all light around $325 \mathrm{~nm}$ is absorbed by the AZB solution. After each time exposure, the solutions were removed from the cells and new solutions were added for a total of 16 AZB samples that were analyzed by UV at $458 \mathrm{~nm}$ for the AZB photoproduct. The results were plotted using the relation \{check symbols; they don't show up on PC\}

$$
\ln \left(1-P / A_{0}\right)=I_{0} \square t / A_{0}
$$

where $\mathrm{A}_{0}$ is the original concentration of $\mathrm{AZB}, \mathrm{P}$ is the concentration of the product 2hydroxyazobenzene (measured by $\mathrm{UV}$ ), $\mathrm{I}_{0}$ is the absorbed radiation intensity in photons/s and $\square$ is the quantum yield (0.04). A plot of $\ln (1-$ $\mathrm{P} / \mathrm{A}_{0}$ ) versus $t$ for each cell gave the linear relations shown in Figure 1 with slopes of $\mathrm{I}_{0} \square / \mathrm{A}_{0}=\mathrm{k}_{\mathrm{p}}$. The relative changes in rate constants $\mathrm{k}_{\mathrm{p}}$ for photolysis from cell to cell compared well with the changes in radiometer values in $\mathrm{mW} / \mathrm{cm}^{2}$ from cell to cell. The values are shown in Table 1.

\section{Photolysis Analysis Module}

SRI developed and constructed a 25cell photolysis analysis module based on the results of a 4-cell prototype. The 25cell design (Figure 3 ) incorporates a vacuum-sealed top plate that should provide the required hermetic sealing and allow for quick loading as it avoids the numerous fasteners that were used in the 4-cell prototype.

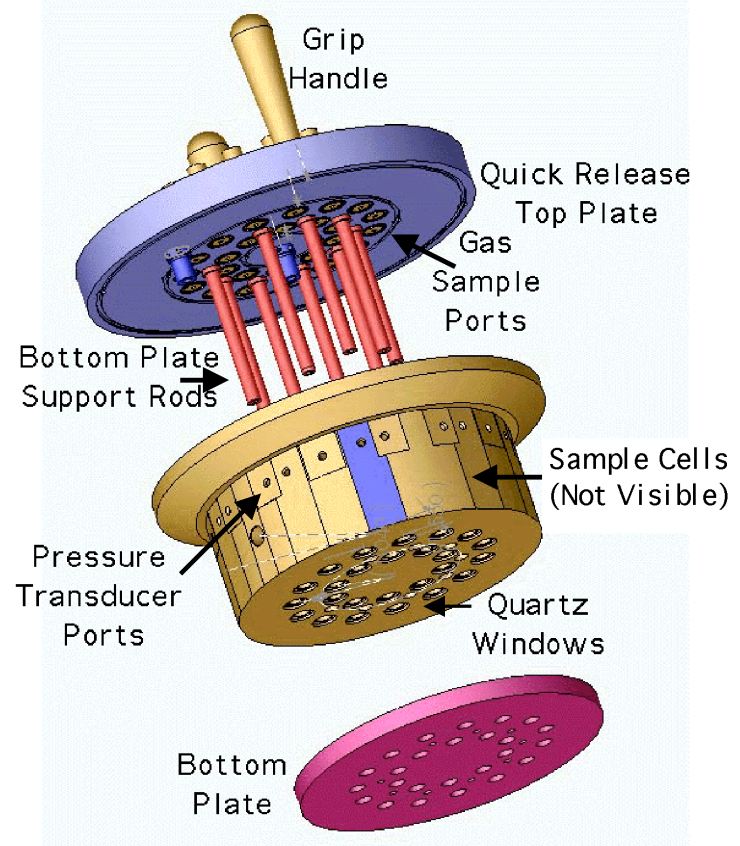

Figure 3. Expanded view of 25-cell analysis module design. 
The dimensions of the analysis module are shown in Figure 4and the footprint is approximately 8 inches in diameter. The photolysis analysis module consists of three pieces, a top quick release plate, a cell body and a bottom plate (Figure 3). The vacuumsealed top plate has tubes that interface the photolysis cells to a septum port for headspace gas analysis. A close-up of the module with the top open is shown in Figure 5. The vacuum seal will facilitate faster and easier access to photolysis cell compartments relative to an earlier design that used a number of screws to seal. As indicated, ports leading from the top of the sample analysis chambers

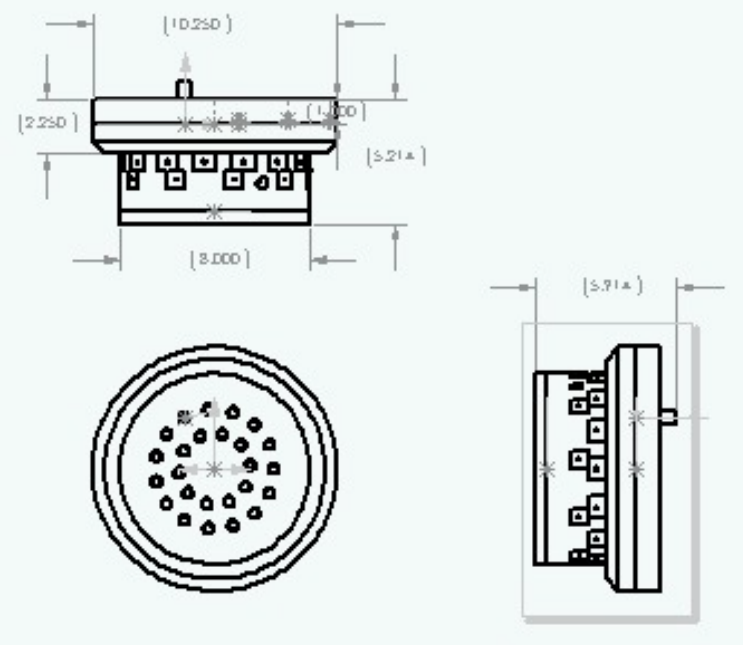

Figure 4. Drawings of 25 cell photolysis module with dimensions in inches.

to the outer wall of the analysis module are designed to allow a line to a pressure transducer array. The bottom plate, which is fixed to the main body of the analysis module via support rods extending from the top of the main body, is used to secure quartz windows to the bottom module. The quartz windows assure that the entire solar spectrum, and into the UV if desired, will be transmitted into the cell.

The pressure transducers are coupled to the photolysis cell via ports that extend from the cell to the side of the cell body. Our design runs a small inner diameter line from the port to a manifold containing the actual pressure transducers so that if a pressure transducer goes out it will be much easier to repair than if the transducer is bonded to the outlet port of the cell body. The pressure is monitored by measuring the voltage response of the pressure transducer as described previously using an in-house developed labview program. The pressure readings can be displayed on a monitor and are saved at user-determined rates ranging from $1 \mathrm{~s}$ to 99

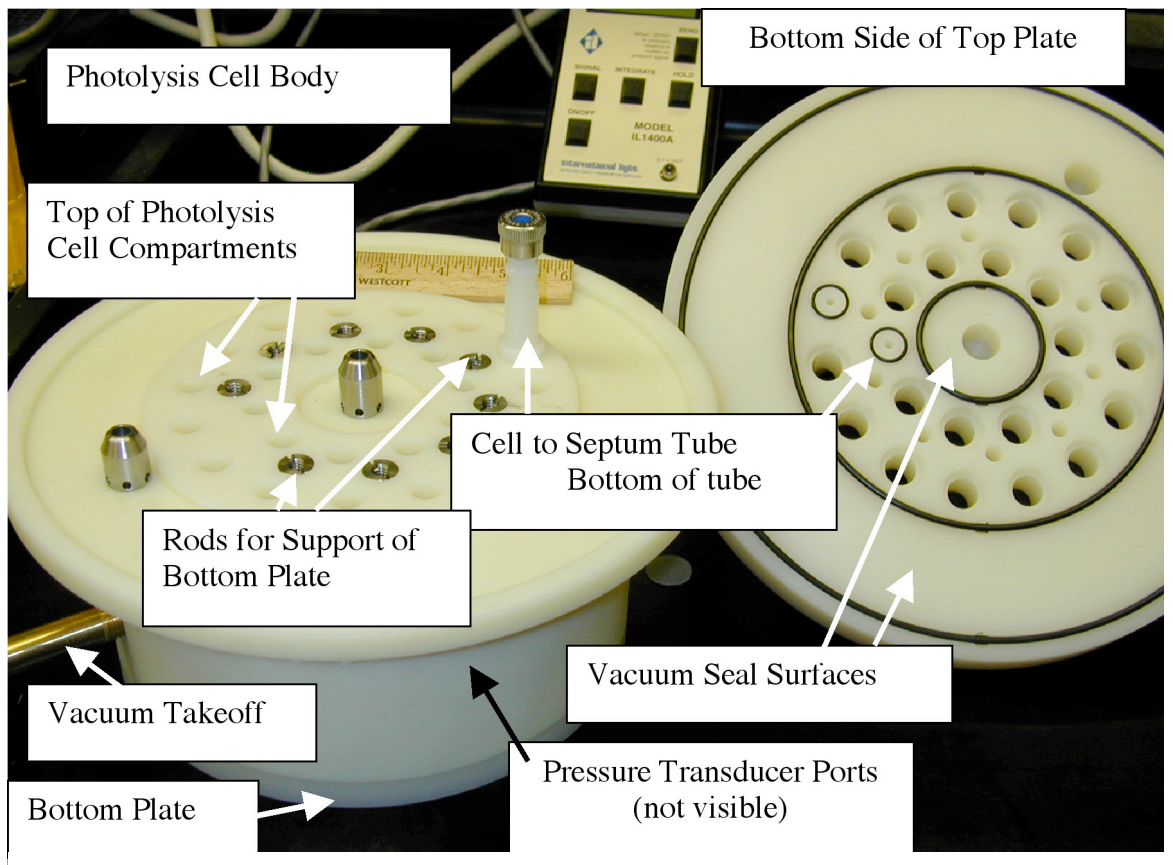

Figure 5. 25-cell photolysis module with top open. min. A picture of the 
photolysis unit in operation is shown in Figure 6

\section{Pressure Measurements}

Pressure measurements were obtained using a commercial pressure transducer. Each sensor is a Wheatstone bridge configuration powered by a constant voltage source $(5 \mathrm{Vdc})$ and produces an output voltage proportional to the inlet pressure. Because of the bridge configuration, the output voltage must be measured using a system with a differential (floating) input. A preliminary voltage measuring workstation has been assembled using a conventional Intel-based PC equipped with an 8-channel (differential), 12-bit, analogto-digital (ADC) converter.

A simple, Windowsbased application was developed to facilitate reading and recording the cell pressures. The application sequentially reads the sensor outputs via the $\mathrm{ADC}$ at a

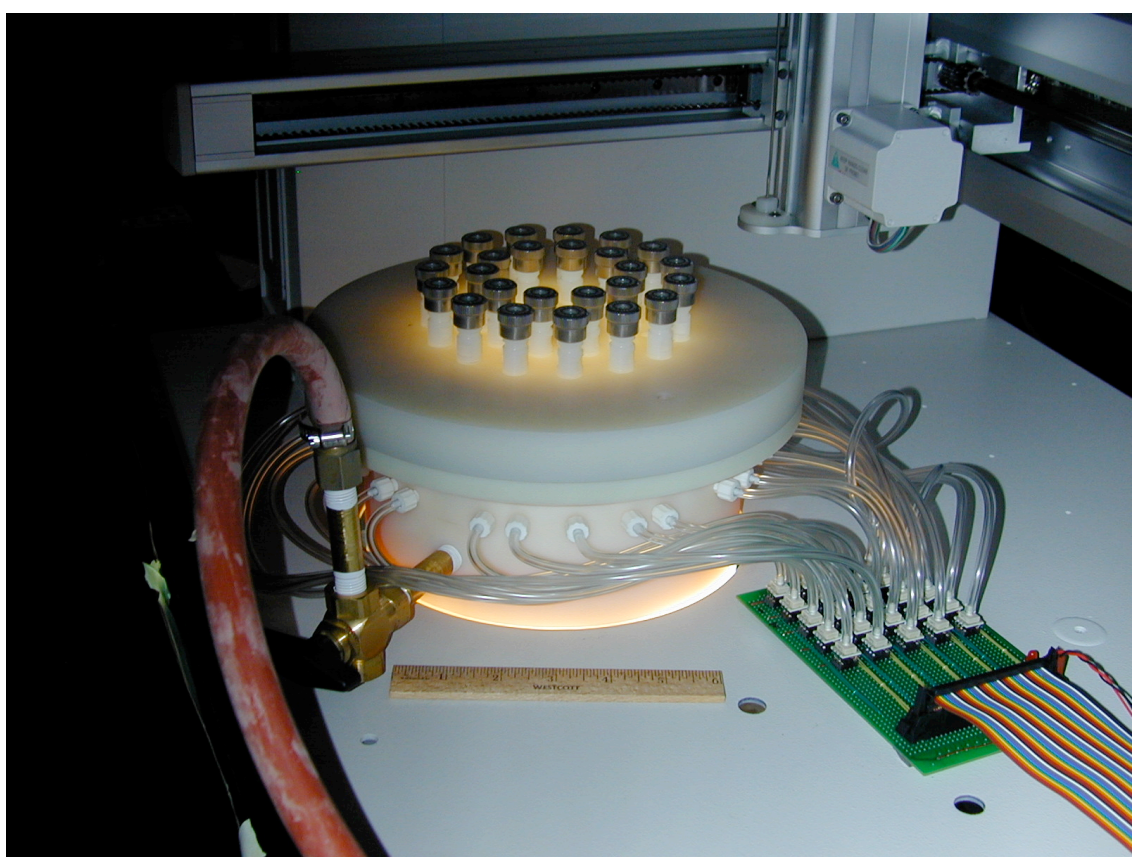

Figure 6. Picture showing 25-cell photolysis module in operation. constant rate of $1 \mathrm{~Hz}$. The data is displayed in absolute pressure units on the computer terminal; however, no data is recorded until the user initiates a run. Before starting a run, the user can select an interval between 1 and 10 seconds at which data will be recorded. The user also has the option of choosing the format in which the data will be saved; either as a tab-delimited text file or inserted directly into an Excel spreadsheet. Once the run begins, the time in seconds and each cell's pressure reading are recorded. Data accumulation continues until manually halted by the user. A sample data set collected during initial testing of

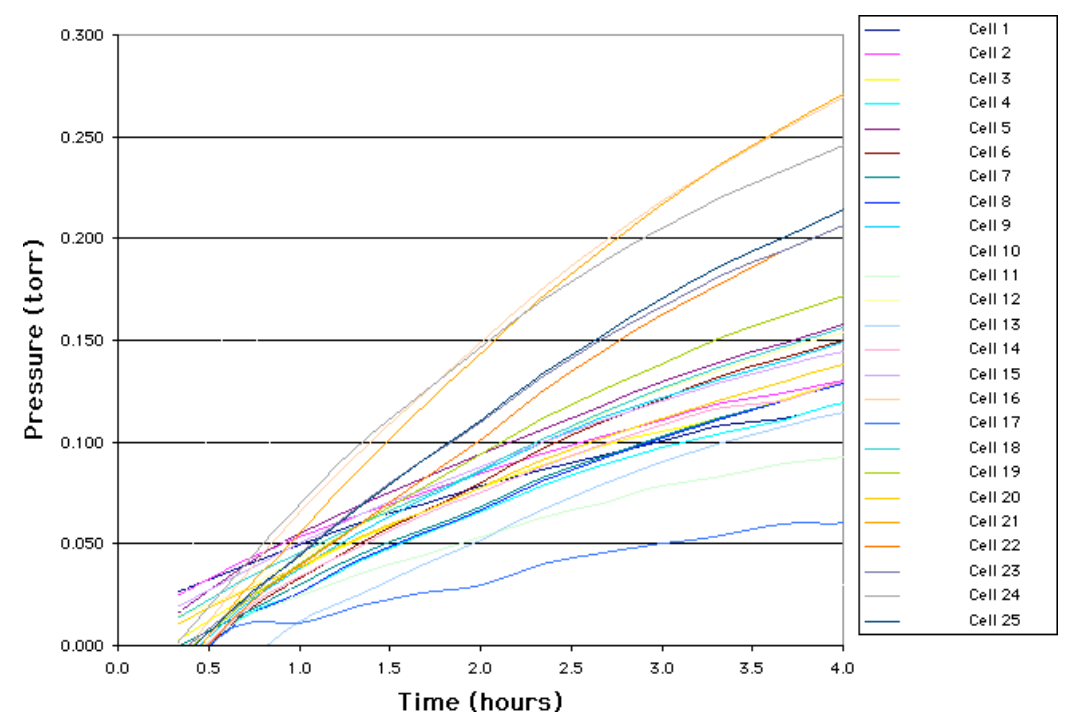

Figure 7. Plot of raw data from 25-cell photolysis module. 
the 25-cell powder analysis module is shown in Figure 7.

Initial leak tests indicated a problem with several of the individual cells. In these experiments the cells were loaded with an arbitrary pressure via a syringe injection through the septum. The pressure was then monitored for 2 hours. A reduction in pressure would indicate a leak.

In the course of this work we observed some drift in the response factor of our pressure transducers. This drift was identified in the course of routine leak testing in which the cells are pressurized and allowed to stand to determine the integrity of each sample cell. As a result response curves for each pressure transducer was determined after every 10 sample runs. This response factor is included in the calculations, along with lamp intensity, used to determine hydrogen yields and ultimately efficiency.

\section{Photocatalysts}

The semiconductor PEC systems explored in this study all consisted of a semiconductor, an electrocatalyst that has been applied to the semiconductor, and an aqueous electrolyte. In the course of this project the primary variable explored in this system was the semiconductor material. There was some investigation into the nature of the electrocatalyst and how it was applied as well as the electrolyte conditions $(\mathrm{pH})$; however, by far the most studied variable was the semiconductor material. The semiconductor materials described below have been divided into $\mathrm{TiO}_{2}$-based and non-titanium-based, with the former being based mostly on nanoparticle materials provided by NanoGram.

The photolysis experiments were conducted in the previously described 25-cell analysis module. In a typical experiment, samples were run in triplicate with two cells containing blanks, for background measurement, and two cells containing a standard photocatalyst, platinized $\mathrm{TiO}_{2}$ (P25, Degussa) as a reference material. The optical density of the nanoparticle suspension samples to be photolyzed was adjusted such that the amount of incident radiation absorbed was between $70-80 \%$. Sample cells are loaded with $10 \mathrm{ml}$ of the test solution, analysis module is sealed, the samples are purged with $\mathrm{N}_{2}$ via a syringe needle and then subjected to the simulated solar spectrum. First screens of test samples were for a period of 16-20 hours, after which the data was collected and analyzed. After subtracting the background pressure and taking into account the pressure transducer response factor and the incident intensity, as discussed earlier, a figure for the volume of hydrogen produced as a function of time is generated. To determine stability, the linearity of the plot of hydrogen production vs. time is investigated. Deviation from linear behavior indicates instability of the photocatalytic system. Because the measurement of the actual amount of incident light that goes into the photocatalyst is difficult to determine, the results of hydrogen production for initial materials investigated are evaluated in terms of their relative activity with respect to the $\mathrm{P} 25$ reference. 
The semiconductor materials explored in this investigation were, for the most part, nanoparticles with dimensions of 100's to 10's of nanometers. The majority of the nanoparticles studied were composed of materials supplied by NanoGram that were then modified at SRI.

\section{$\mathrm{TiO}_{2}$-Based Photocatalysts}

NanoGram utilizes lasers in its production of inorganic, crystalline nanomaterials. The process is depicted in Figure 8, and a close-up of the reaction chamber is shown in Figure 9. The NanoGram process for producing nanoparticles, $\mathrm{NPM}^{\mathrm{TM}}$, is based on a laser-driven chemical reaction that takes place within a well-controlled reaction zone. $\mathrm{A} \mathrm{CO}_{2}$ laser beam provides a heat source for rapid heating (at a rate on the order of $10^{5} \mathrm{~K} / \mathrm{s}$ ) within a tiny and controlled reaction zone, resulting in hightemperature pyrolysis and chemical reaction between the precursors. Immediately after the laser reaction zone, nanoscale particles are formed due to a rapid cooling (at a rate on the order of $10^{5} \mathrm{~K} / \mathrm{s}$ ) without any contact with a cooling agent. This process occurs essentially at nonequilibrium.

In contrast to conventional processes, which either grind large particles into small particles or use

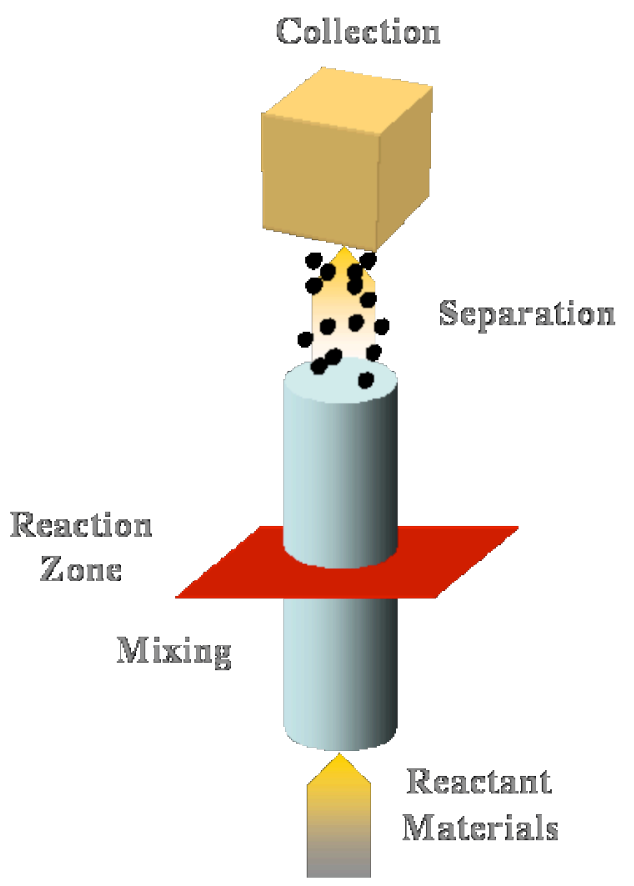

Figure 8. NanoGram laser pyrolysis process. poorly controlled reaction vessels, NanoGram virtually builds nano-scale particles from the atomic level. This patented method is the best process for manufacturing nanomaterials as measured by quality, purity, composition control, particle-size control, and cost effectiveness. The company can produce a wide range of crystalline nanomaterials including oxides, carbides, sulfides, nitrides, phosphides, and silicates, as well as complex materials made of three or four (or more) elements, called ternary and quaternary materials. The process can produce the widest range of endproduct compounds and utilize a full complement of lower cost, commodity chemicals as feedstocks.

NanoGram has developed an efficient chemical reaction process inside a chamber by optimizing an

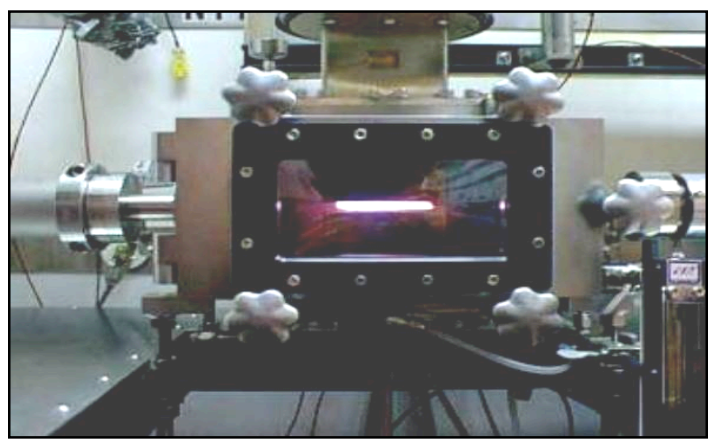

Figure 9. Close-up of NanoGram laser pyrolysis reactor. equipment design and a flow control of precursors and particle collection. As a result, throughputs up to $1 \mathrm{~kg} /$ hour of production for several test compounds have been demonstrated. Process scale-up work is ongoing with substantial results 
achieved already. Current process design modifications are expected to deliver another throughput increase in the range of 10-20X (to approximately $4 \mathrm{~kg} / \mathrm{hr}$ ).

The materials provided by NanoGram were, for the most part, titania samples with different particle sizes, degrees of crystallinity and phase (rutile, anatase and mixtures of both). Some of the data collected using $\mathrm{TiO}_{2}$ samples provided by NanoGram is shown in Table 2. The letters $\mathrm{R}$, for rutile, and A, for anatase, in the sample name refer to the phase of the sample, and when both are present it indicates a mixed-phase material with the predominant phase being the first letter. The data series shown looked at the $\mathrm{TiO}_{2}$ samples with three different methods of electrocatalyst application. Photo Pt is the photoreduction of Pt from a solution containing Pt ions followed by heat treatment under reducing conditions, and is the most common Pt deposition technique. IW Pt refers to Pt deposited using an incipient wetness and involves evaporation of a Pt salt onto the particle surface followed by drying and then heat treatment. PtRuIrOs is a photocatalyst that was applied in a similar fashion as the IW Pt electrocatalyst. As the data shows there is some difference in the hydrogen production rate in some samples, however in general this is very small. As such we decided to look solely at samples with the Photo Pt electrocatalyst deposition.

Table 2. Selected Data (standard deviation in parenthesis) from Photolysis Experiments of $\mathrm{Various} \mathrm{TiO}_{2}$ Samples.

\begin{tabular}{|c|c|c|c|c|}
\hline Sample & $\begin{array}{c}\text { median } \\
\text { diameter, } \mu \mathrm{m}\end{array}$ & $\begin{array}{c}\mathrm{uL} \mathrm{H}_{2} / \mathrm{hr} \mathrm{m}^{2} \\
\text { Photo Pt }\end{array}$ & $\begin{array}{c}\text { uL } \mathrm{H}_{2} / \mathrm{hr} \mathrm{m}^{2} \\
\text { IW Pt }\end{array}$ & $\begin{array}{c}\mathrm{uL} \mathrm{H}_{2} / \mathrm{hr} \mathrm{m}^{2} \\
\text { PtRulrOs }\end{array}$ \\
\hline A 25 & 0.11 & $27(3.0)$ & $34(7.2)$ & $21(3.8)$ \\
\hline A 33 & 0.10 & $25(4.2)$ & $44(7.3)$ & $23(5.8)$ \\
\hline A 30 & 0.08 & $32(5.9)$ & $56(9.2)$ & 31 (4.9) \\
\hline AR 101 & 0.08 & $42(6.4)$ & $63(10.2)$ & $58(11.2)$ \\
\hline RA 37 & 0.07 & $28(5.8)$ & $36(8.1)$ & $23(6.3)$ \\
\hline AR 51 & 0.07 & $48(6.2)$ & $64(11.8)$ & $67(10.5)$ \\
\hline RA 76 & 0.07 & $21(3.9)$ & $24(5.3)$ & $25(8.2)$ \\
\hline A 50 & 0.06 & $38(7.2)$ & $44(8.9)$ & $34(7.3)$ \\
\hline A 55 & 0.06 & $36(5.1)$ & $38(8.4)$ & $31(7.9)$ \\
\hline AR 110 & 0.06 & $29(6.2)$ & 68 (12.9) & $71(13.1)$ \\
\hline A 24 & 0.05 & $24(5.8)$ & $32(7.5)$ & $41(8.9)$ \\
\hline A 57 & 0.04 & $33(7.1)$ & $41(7.3)$ & $37(7.4)$ \\
\hline AR 113 & $<0.03$ & $46(6.4)$ & 69 (14.1) & $71(11.8)$ \\
\hline A 82 & $<0.03$ & $35(7.3)$ & $41(7.5)$ & $33(6.8)$ \\
\hline P25 & 0.09 & $21(6.5)$ & $29(6.2)$ & $34(8.3)$ \\
\hline
\end{tabular}



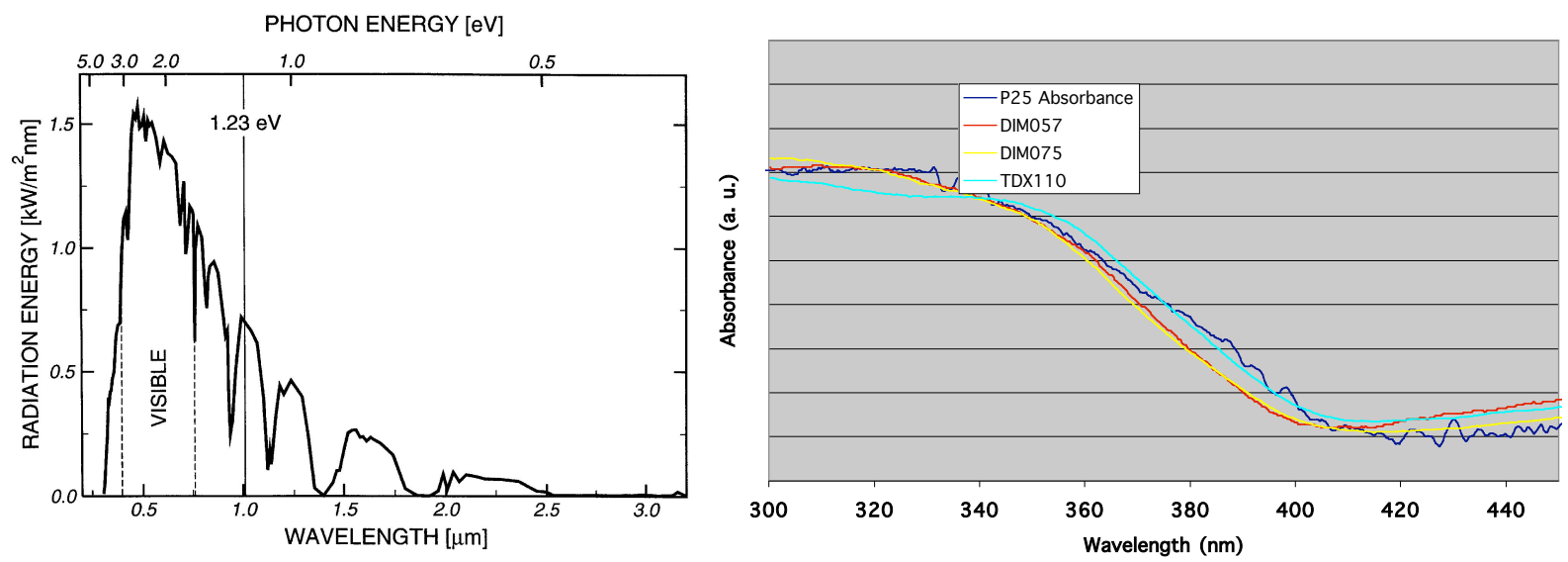

Figure 10. Solar spectrum (left) $(1 \square \mathrm{m}=1,000 \mathrm{~nm})$, and absorbance spectrum (right) of several titania materials.

One of the main problems with the titanium oxide materials is that they do not absorb sufficient solar radiation due to their large band gap, $>3.1 \mathrm{eV}$. Figure 10 shows the solar and absorbance spectra of titania. On the other hand, the titania materials are extremely stable, which is a very desirable feature. As such, we focuse our efforts on doping the titania in an attempt to lower the band gap and hence increase the amount of visible light absorbed, while maintaining photostanility.

We prepared a series of TiON materials by the hydrolysis reaction of Ti(isopropoxide) and diethylamine, similar to that reported by Chen and Burda, [Chen and Burda J. Phys Chem B., $108,15446,2004]$. Another series of TiON samples were prepared by the reaction of NanoGram's $\mathrm{TiO}_{2}$ nanoparticles with ammonia at temperatures from $400^{\circ} \mathrm{C}$ to $600^{\circ} \mathrm{C}$, similar to the procedure reported by Diwald et al. [Diwald et al., J. Phys. Chem. B., 108, 52, 2004]. All particle sets were platinized at $2 \%$ using the previously described photo-decomposition method. The results with the materials made with the NanoGram materials had a somewhat improved performance relative to what has been reported using $\mathrm{Ti}$ (isopropoxide) as the $\mathrm{Ti}$ source. However the overall performance of all of the TiON materials studied was well short of that required for costeffective PEC hydrogen production.

In summary, none of the titaniumbased semiconductor materials evaluated exhibited efficiencies $>0.5 \%$ solar to hydrogen conversion based on their performance relative to the $\mathrm{P} 25$ reference. Therefore, we did not perform a detailed analysis on any of these materials, but

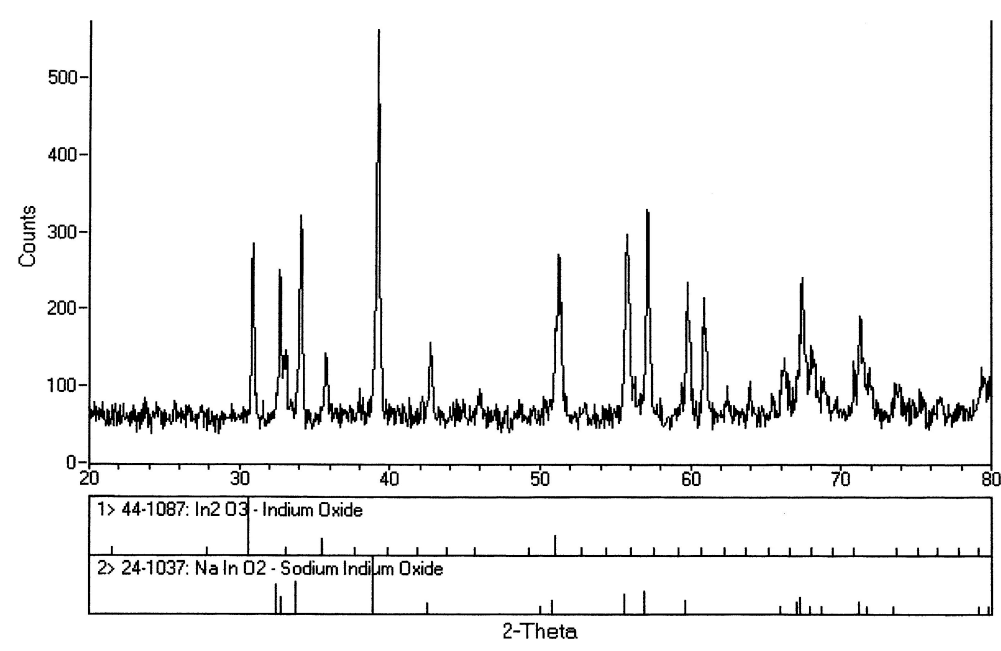

Figure 11. XRD pattern indicating formation of a mixed alkali $(\mathrm{Na} / \mathrm{K})$ indium oxide. 
instead considered alternative semiconductors. On a positive note, the stabilities of most of the titanium-based semiconductors was excellent, with very few showing any measurable decrease in the hydrogen production rates over $18-20 \mathrm{~h}$ exposures.

\section{Non-Titanium-Based Photocatalysts}

After exploring titanium oxide materials and determining that they are inappropriate for use in an economical PEC hydrogen production system, we turned to

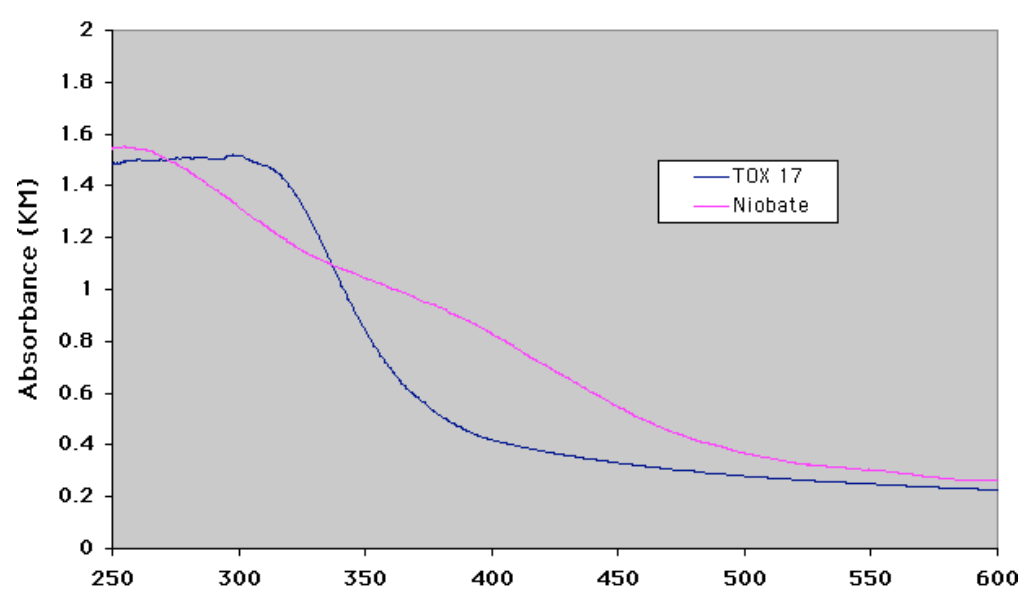

Figure 12. Absorbance spectrum of a niobate compared to a titania. non-titanium materials. The majority of the materials examined were oxides that, as in the titania materials, were doped to decrease the band gap and increase the amount of the solar spectrum the material absorbs. These materials included some mixed alkali ( $\mathrm{Na}$ and $\mathrm{K}$ ) indium oxides, the $\mathrm{x}$ ray diffraction (XRD) for one of which is shown in Figure 11, and barium niobium oxide with and without cobalt substitution for niobium (Figure 12). These materials have shown promise in the literature and in some modeling experiments also reported in the literature. However, none of the single phase materials we investigated showed efficiencies and stabilities that would warrant further investigation as the semiconductor component of a PEC hydrogen production system.

Another material of interest is $(\operatorname{AgIn})_{x} \mathrm{Zn}_{(1-}$ ${ }_{\mathrm{x}} \mathrm{S}$, as it is reported to exist as discrete AgIn and $\mathrm{ZnS} n$ and p-type phases. This is reported to result in discrete $\mathrm{p}-\mathrm{n}$ junctions being formed in the material, which should improve charge separation efficiencies relative to a singlephase material. The materials were prepared in a similar fashion as that reported by Tsuji et al. [J. Am. Chem. Soc. 2004, 126, 13406-13413] with some modification to the stoichiometries. The XRD and UV-Vis reflectance spectra are shown in Figure 13. As the UV-Vis absorption spectrum indicates, a band gap of approximately $800 \mathrm{~nm}(1.5 \mathrm{eV})$. XRD is consistent with its reported structure. The onset of optical absorption changes very little with respect to the value of $\mathrm{x}$, while some variation in the XRD is observed. The yields
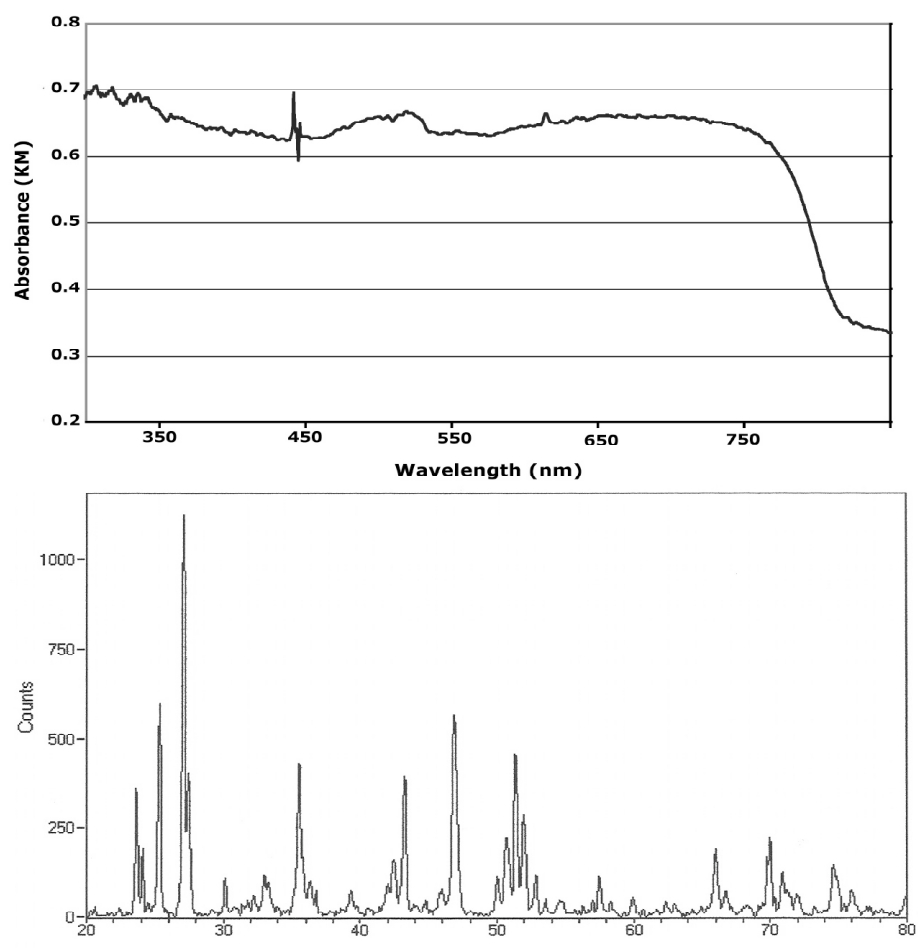

Figure 13. UV-Vis spectrum and XRD of $(\mathrm{AgIn})_{0.2} \mathrm{ZnS}$. 
of hydrogen indicate that this materials is approximately 20 times more efficient than the P25 reference material; however, this is still about an order of magnitude below the goal of $10 \%$ solar efficiency, and these materials did show a decrease in the hydrogen production rate after 16-20 hours. Nonetheless, this material does show some promise and perhaps should be investigated in more detail.

The final synthetic work focused on the growth of InP nanorods on conductive substrate surfaces (ITO or FTO) using protocols established in the literature [Kan,Y Assaf Aharoni, Taleb Mokari and Uri Banin, Faraday Discuss. 125, 23-38, 2004; and Yujie Xiong, Yi Xie, Zhengquan Li, Xiaoxu Li, and Shanmin Gao, Chemistry - A European Journal, 10 (3), 654-660, 2004]. Our attempts were not successful, we believe because we did not generate an adequate array of the gold nanocatalyst on which the InP rods are grown.

In summary, this work indicates that a single-phase material will probably not have adequate solar-to-hydrogen efficiencies to be a component of a PEC hydrogen production system. The mixed-phase materials, while still inadequate for economical production, do show improvements and should be further investigated. Of significant promise are the modeling results, which indicate that efficiencies and photostability can be improved by engineering coreshell nanostructures. Although we were unable to validate these models, the synthesis of nanostructures is advancing rapidly, and the author is optimistic that nanostructures can be constructed that will validate the models we generated and lead to commercially viable PEC hydrogen production.

\section{Modeling}

We modeled two potential nanostructures to assess their potential use in PEC hydrogen production. One involved the modeling of a nanostructured ferroelectric semiconductor, and the other investigated the modeling of core-shell nanostructures.

\section{Nanostructured Ferroelectric Modeling}

Recently Kato et al. discovered that when $\mathrm{NaTaO}_{3}$ is doped by a few percent lanthanum, a zigzag surface nanostructure with step size 3-15 nm appears [J. Am. Chem. Soc. 125, 581 (2001)]. They also found that the edges in this zigzag structure have excess positive charges $(p$ regions), whereas the grooves have excess negative charges ( $n$-regions). The alternating $p$ - and $n$-regions on the surface facilitate fast separation of electrons and holes and greatly enhance the hydrogen-production efficiency. To date it is not understood at all why and how such surface nanostructures are formed. We have tried to provide an understanding and rationale of the formation of these surface structures from a theoretical point of view.

First, we notice from literature that $\mathrm{NaTaO}_{3}$ was claimed to be ferroelectric [Matthias, Phys. Rev. 75, 1771 (1949)], meaning it has a dielectric polarization even in the absence of an electric 
field. Although this claim was challenged later [Smolenskii, Zhur. Tekh. Fiz. 27, 2528 (1957)], it is fair to say that $\mathrm{NaTaO}_{3}$ tends to have a strong dielectric polarization under perturbations such as electric fields, strains, and doping, which is supported by that fact that both $\mathrm{KTaO}_{3}$ and $\mathrm{Na}_{\mathrm{x}} \mathrm{K}_{1-\mathrm{x}} \mathrm{TaO}_{3}$ are ferroelectric.

Second, we carried out a first-principles calculation to determine whether La will substitute $\mathrm{Na}$ or $\mathrm{Ta}$ in La-doped $\mathrm{NaTaO}_{3}$. We considered a supercell with $2 \times 2 \times 2$ unit cells (the corresponding doping concentration is $1 / 8$ ) and compared the energies of configurations with La in different locations at fully relaxed geometry. We found an energy difference of $16.5 \mathrm{eV}$ between the two configurations, indicating that it is energetically favorable for La to substitute $\mathrm{Na}$. Since a stable La ion has three positive charges, the substitution of $\mathrm{La3}+$ ions for $\mathrm{Na}+$ ions will undoubtedly create a strong electric field on nearby $\mathrm{Ta}$ and $\mathrm{O}$ ions, producing a further polarization. We also found that La doping also induces considerable strains in $\mathrm{NaTaO}_{3}$. Thus, these theoretical calculations indicate that a La-doped $\mathrm{NaTaO}_{3}$ likely has a macroscopic dielectric polarization.

Third, a ferroelectric material with strains and/or impurities tends to form domains with different polarization directions. According to a theory by Zhirnov [Sov. Phys. JETP. 35, 822 (1959)], the thickness of the transition region between two $90^{\circ}$ domains in $\mathrm{BaTiO}_{3}$ is about 5-10 $\mathrm{nm}$. The thickness depends on strain parameters, and it is not unreasonable to assume that the strain parameters in $\mathrm{NaTaO}_{3}$ would be similar to those in $\mathrm{BaTiO}_{3}$ (they have the same crystal symmetry). Thus we think that the observed step size of 3-15 nm in La-doped $\mathrm{NaTaO}_{3}$ reflects the length scale of ferroelectric domain structures in the material.

\section{Core-Shell Nanostructure Modeling}

This work was inspired by reports that indicated that photogenerated charge carriers in coreshell nanoparticles, depending on the configuration, can be separated and spatially isolated in either the core or shell [Kortan, A. R.; Hull, R.; Opila, R. L.; Bawendi, M. G.; Steigerwald, M. L.; Carroll, P. J.; Brus, L. E. J. Am. Chem. Soc. 1990, 1327,1990 and L. P. Balet, S. A. Ivanov, A. Piryatinski, M. Achermann, and V. I. Klimov, NanoLetters, 2004, 4, 1485.]. This results in extremely efficient charge separation, which would be a means to improve catalyst efficiency in a PEC hydrogen production system. However, the core-shell structures described in the literature result in isolation of one of the charge carriers in the core, making it unavailable for water splitting. As such we have modified the core-shell structure from a spherical one to a rod-based structure, which allows use of photogenerated charge carries and potentially leads to an improved system for PEC hydrogen production. We exploit quantum confinement as well as its interaction with strains in coaxial cylindrical core-shell nano-rods, see figure 14 for dimension definitions, to effectively tailor their electronic structures. 
This work has been published in a peerreviewed publication [Yu Z.G., Pryor C.E., Lau W.H., Berding M.A. and MacQueen D.B., J. Phys. Chem. B 109, 22913-22919] and a copy is attached as Appendix A. In a
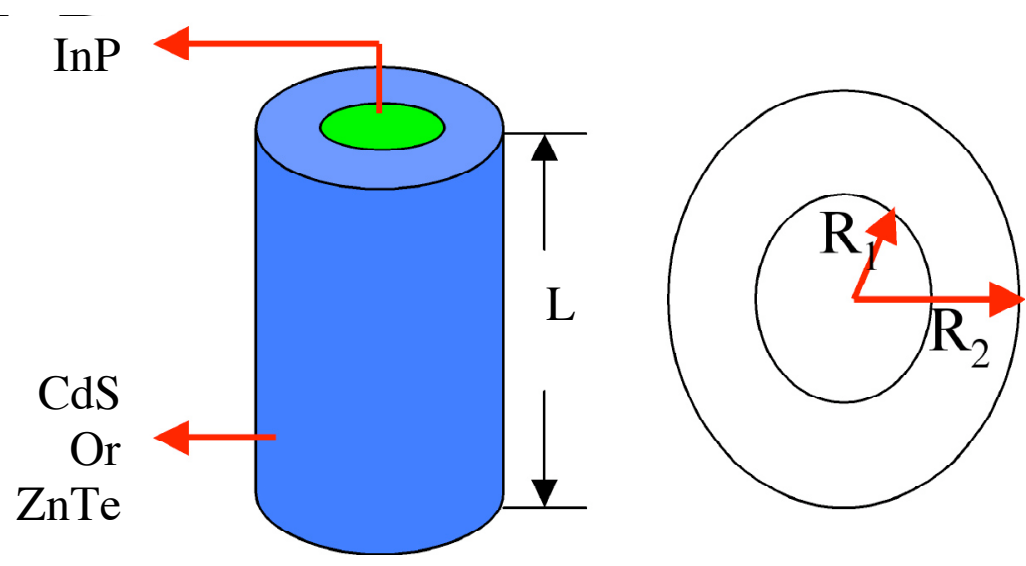
semiconductor nanoheterostructure, the inevitable lattice mismatch between

Figure 14. Core-shell nano-rod strucures indicating dimensuion definitions

different semiconductors gives rise to a significant strain and accordingly, elastic energy. In modeling these nanostructures, it is important to consistently describe the strain and its effects on the electronic structure.

To identify suitable semiconductor nanostructures for efficient hydrogen production, we studied electronic levels and wave functions of various semiconductor nano-heterostructures using a strain-dependent k.p approach. This approach was developed by Craig and collaborators [C. Pryor, Phys. Rev. B 57, 7190 (1998)] and has been successfully applied to different quantum dots.

The strain in a nanostructure is computed by minimizing the free energy for a cubic crystal

$$
\begin{aligned}
& F=\square l^{3} x \frac{1}{2} C_{x x x x}\left(\square_{x x}^{2}+\square_{y y}^{2}+\square_{z z}^{2}\right)+C_{x x y y}\left(\square_{x x} \square_{y y}+\square_{x x} \square_{z z}+\square_{y y} \square_{z z}\right) \\
& +2 C_{x y x y}\left(\square_{x y}^{2}+\square_{x z}^{2}+\square_{y z}^{2}\right) \square \square\left(\square_{x x}+\square_{y y}+\square_{z z}\right)
\end{aligned}
$$

where $\square_{\mathrm{ij}}$ is the strain tensor, the C's are material dependent elastic constants, and $\square$ is a parameter used to enforce the lattice mismatch between two materials. The strain is given in terms of the displacement by $\square_{i j}=\left(\partial_{i} u_{j}+\partial_{j} u_{i}\right) / 2$. F is constructed as a function of $u_{i}$ 's on a cubic grid with derivatives replaced by difference, and then is minimized using the conjugated gradient algorithm. The grid box that contains the structure of interest is chosen sufficiently large to ensure that the boundary effect is negligible. 
Because the materials lack inversion symmetry, the strain produces a polarization given by $P_{i}=e_{i j k} \square_{j k}$, leading to an additional electrostatic potential. For cubic semiconductors, the only nonzero elements of the piezoelectric tensor are $e_{x y z}=e_{z x y}$ $=e_{y z x} \equiv e_{14}$. From the polarization, it is straightforward to compute the electrostatic potential $\mathrm{V}_{\mathrm{p}}$ by numerically solving the Poisson equation.

The electronic structure is calculated in the envelope approximation using $\square_{i j}$ and $V_{p}$ computed above. The electron Hamiltonian is

$$
H=H_{k}+H_{s}
$$

The explicit expressions of $H_{k}$ and $H_{s}$ (8x8 matrices) can be found in [C. Pryor, Phys. Rev. B 57, 7190 (1998)]. The values used for the various material parameters in the Hamiltonian were taken from direct measurements, which have been documented in literature.

As an example of the modeling results we consider a core/shell spherical structure of $\mathrm{InP} / \mathrm{ZnSe}$ with $\mathrm{R}_{1}=2.5 \mathrm{~nm}$ and $\mathrm{R}_{2}=5.0 \mathrm{~nm}$. The obtained band alignment is shown in Figure 15. We see that the core/shell InP/ZnSe structure has a type-II band alignment, namely that the bottom of the conduction band and the top of the valence band are located in different regions. Consequently, a photo-excited electron-hole pair created in the InP region will dissolve with the electron localized in the core (InP) and the hole in the shell ( $\mathrm{ZnSe}$ ). We plot in Figure 16 the 3-dimensional spatial distribution of wave functions of the lowest-energy level in the conduction band (LUMO) and the highest-energy level in the 
valence band (LUMO). We s shell.

In another example we 1 the energies of the HOMO a fixed height $H=2 \mathrm{~nm}$ and ov' in the core $\left(P_{\text {core }}>0.5\right)$ or in $\mathrm{t}$ the LUMO are well spatially LUMO in the shell. With inc to the core, and eventually $b_{1}$ core radius increases from $R$ $\mathrm{eV}$, and the LUMO changes satisfy the overlap requirer

The envisioned device structure based on the core-shell InPCdS or InP-ZnSe

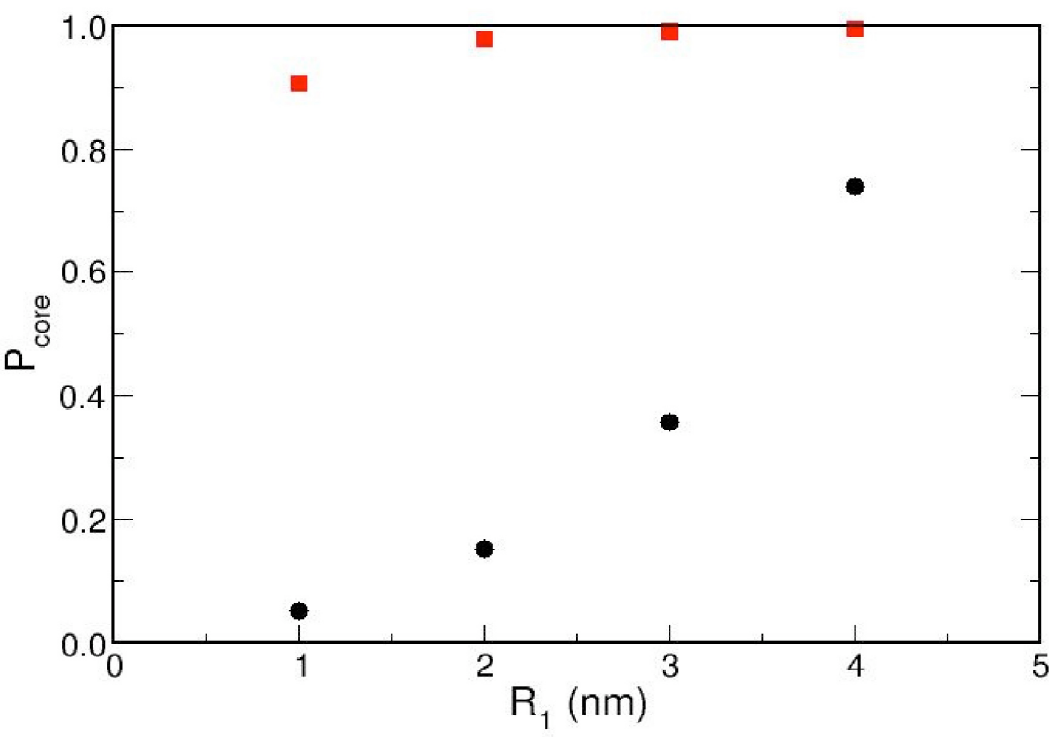

Figure 17. Integrated charge distribution in core for the HOMO (squares) and the LUMO (circles) as a function of R1 in core-shell InP-CdS nanorods with fixed $R=5 \mathrm{~nm}$ and $\mathrm{L}=2 \mathrm{~nm}$.

nanorods for hydrogen

production are illustrated in Fig. 18. In this structure, InP is protected from corrosion in water by the large-gap CdS. The electron (LUMO) and the hole (HOMO) of a photoexcited electron-hole pair are spatially separated with the former in the shell and the latter in the core, leading to faster and more efficient charge transfer. The energies of the HOMO and LUMO can be shifted upward or downward to overlap the water redox potentials. Thus, all the aforementioned criteria are satisfied.

In summary we find that core-shell InP-CdS and InP-ZnTe nano-rods, where both energies and wave-function distributions of electrons and holes can be tailored to a considerable extent, can meet all criteria and therefore serve as promising photocatalysts for PEC hydrogen production. 
(a)
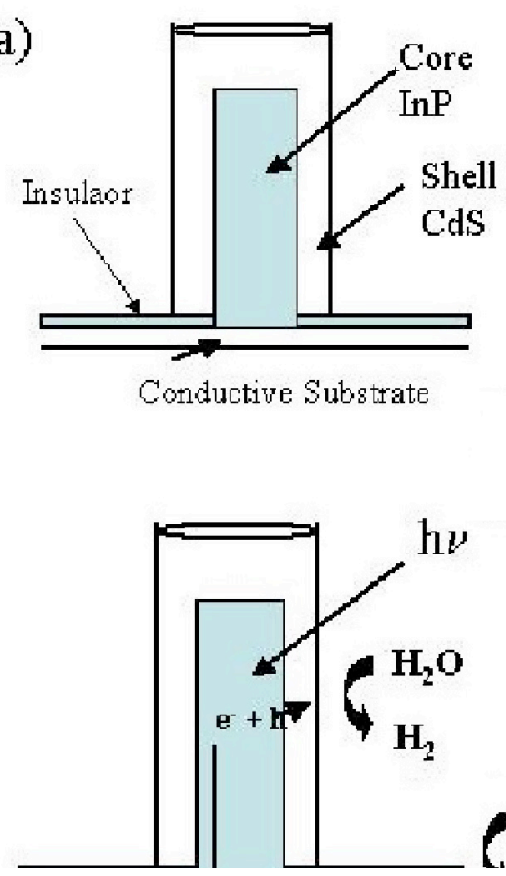

(b)

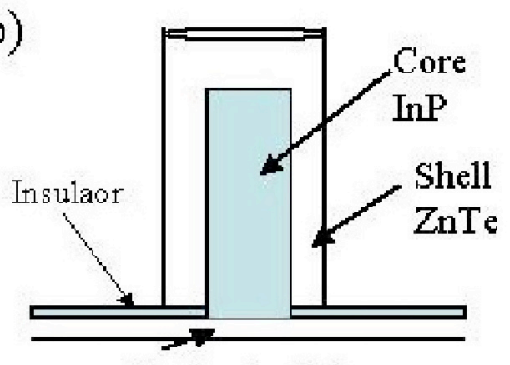

Conductive Substrate

Figure 18. Suggested nano-rod core-shell structures and mechanism of charge separation

\section{Product Development and Technology Transfer}

\section{Publications and Presentations}

The following publication has been published and is included as Appendix A. Several manuscripts are in preparation and will be submitted to DOE upon completion and acceptance for publication.

Yu Z.G., Pryor C. E., Lau W.H., Berding M.A., and MacQueen D.B. J. Phys. Chem. B 109, 22913-22919.

Other than the annual review, the following presentations have been given:

Global Climate and Energy Project Hydrogen Workshop, April 14 and 15, 2003, Stanford University, Palo Alto, CA.

American Ceramics Society, 55th Pacific Coast Regional \& Basic Sciences Division, Oakland, CA October 19-22, 2003.

NanoSig Clean Energy \& Nano Catalyst Conference, Menlo Park, CA, August 20, 2004. 


\section{Web Sites}

None

\section{Networks and Collaborations}

We collaborated with Professor Jawwad Darr, Queen Mary University, London, on an EPSCOR-funded effort to develop a high-throughput photolysis analysis station for the purpose of high-throughput analysis of the photocatalytic activity of materials generated with a continuous hydrothermal synthesis apparatus. Dr. MacQueen's role is as a consultant assisting with the design and construction of the high-throughput photolysis analysis station. This 3-year effort began in July 2006.

\section{Technologies and Techniques}

Most of the technologies and techniques used in this project were already in practice. This effort did combine some of these technologies, such as pressure transducers to monitor hydrogen production and the development of a 25 cell photolysis module (as opposed to doing one sample at a time), in ways that could be considered unique.

Inventions/Patent Applications, Licensing Agreements

None

Other

None 